\title{
Transposons and satellite DNA: on the origin of the major satellite DNA family in the Chenopodium genome
}

\author{
Alexander Belyayev ${ }^{1 *} \mathbb{D}$, Jiřina Josefiová', Michaela Jandová', Václav Mahelka ${ }^{1}, K^{\prime}$ Karol Krak ${ }^{1,2}$ and Bohumil Mandák ${ }^{1,2}$
}

\begin{abstract}
Extensive and complex links exist between transposable elements (TEs) and satellite DNA (satDNA), which are the two largest fractions of eukaryotic genome. These relationships have a crucial effect on genome structure, function and evolution. Here, we report a novel case of mutual relationships between TEs and satDNA. In the genomes of Chenopodium s. str. species, the deletion derivatives of thp2 conserved domain of the newly discovered CACTA-like TE Jozin are involved in generating monomers of the most abundant satDNA family of the Chenopodium satellitome. The analysis of the relative positions of satDNA and different TEs utilizing assembled Illumina reads revealed several associations between satDNA arrays and the transposases of putative CACTA-like elements when an $\sim 40$ bp fragment of tnp2 served as the start monomer of the satDNA array. The high degree of identity of the consensus satDNA monomers of the investigated species and the tnp2 fragment (from 82.1 to 94.9\%) provides evidence of the genesis of CficCl-61-40 satDNA family monomers from analogous regions of their respective parental elements. The results were confirmed via molecular genetic methods and Oxford Nanopore sequencing. The discovered phenomenon leads to the continuous replenishment of species genomes with new identical satDNA monomers, which in turn may increase species satellitomes similarity.
\end{abstract}

Keywords: CACTA transposons, Satellite DNA, Transposase, Chenopodium, Next generation sequencing, Oxford Nanopore sequencing

\section{Introduction}

Transposable elements (TEs) and satellite DNA (satDNA) are the major components of the eukaryotic genome repeatome, accounting for up to $90 \%$ of plant nuclear DNA [1]. Among the internal sources of genotypic change, TEs can be considered the most powerful due to their ability to move, insert at novel locations and thereby shape and induce the specialization of the landscapes of coding and non-coding DNA fractions [2-4]. In contrast, satDNA, which consists of long, latereplicating, noncoding arrays of tandemly arranged

\footnotetext{
* Correspondence: alexander.belyayev@ibot.cas.cz

${ }^{1}$ Czech Academy of Sciences, Institute of Botany, Zámek 1, CZ-252 43 Průhonice, Czech Republic

Full list of author information is available at the end of the article
}

monomers and is predominantly concentrated in the heterochromatic regions of chromosomes [5, 6], is nonmobile, although recent studies suggest its involvement in various functions ranging from chromosome organization and pairing to cell metabolism and the modulation of gene functions [1, 7-10].

The relationships between these two large fractions are fascinating. Extensive and complex links between TEs and satDNAs exist in eukaryotic genome, creating a complex network of sequences that has a crucial effect on genome structure, function and evolution [10]. There is growing evidence of the involvement of TEs in generating a library of tandem repeats that can be dispersed throughout the genome and, in some cases, amplified into long arrays of new satDNAs [10-12]. Recently, the

\section{$\triangle B M C$}

(C) The Author(s). 2020 Open Access This article is licensed under a Creative Commons Attribution 4.0 International License, which permits use, sharing, adaptation, distribution and reproduction in any medium or format, as long as you give appropriate credit to the original author(s) and the source, provide a link to the Creative Commons licence, and indicate if changes were made. The images or other third party material in this article are included in the article's Creative Commons licence, unless indicated otherwise in a credit line to the material. If material is not included in the article's Creative Commons licence and your intended use is not permitted by statutory regulation or exceeds the permitted use, you will need to obtain permission directly from the copyright holder. To view a copy of this licence, visit http://creativecommons.org/licenses/by/4.0/. The Creative Commons Public Domain Dedication waiver (http://creativecommons.org/publicdomain/zero/1.0/) applies to the data made available in this article, unless otherwise stated in a credit line to the data. 
possibility of elucidating the details of this process by using next-generation sequencing (NGS) technology has arisen through the comparative analysis of entire species repeatomes.

Genome assemblies resulting from the alignment and merging of short DNA fragments to reconstruct the original sequence allow the determination of the interposition of genome elements and, to a certain extent, the possible links between them. For the analysis of the interrelationships between TEs and satDNA, we utilized the genomes of Chenopodium s. str. (also referred to as the Chenopodium album aggregate). Species of the $C$. album aggregate are distributed worldwide, with the highest species diversity in temperate areas [13]. The majority of the species in this diploid-polyploid complex are phenotypically exceptionally plastic [14] and are able to grow under a wide range of conditions [15]. Eight monophyletic lineages have been recognized within the Eurasian representatives of this group [16]. Five of these clades are represented by extant diploid species, whereas the remaining three were reconstructed based on the sequences found in polyploid taxa and are considered to have originated from extinct or still unknown species. All tetraploid taxa were found to be of allopolyploid origin arising as a result of hybridization between diploids from different clades. The allohexaploids exhibit a combined tetraploid and diploid genome (for the genomic composition of the analyzed taxa, see Table 1). The evolutionary history of this typical angiosperm group revealed by key molecular-phylogenetic markers can be briefly described as follows: the early differentiation of the $C$. album aggregate coincided with the beginning of the Miocene Climatic Optimum 20 Mya. Clade H separated upon the transition between the Serravallian and Tortonian Ages, 11 Mya. However, the main lineages were formed in the Pliocene. Subsequent speciation within the lineages and the appearance of the majority of polyploids took place in the Quaternary Period [16].

The main characteristic of the Chenopodium species satellitome is the presence of a basic repeat unit of approximately $40 \mathrm{bp}$ (CficCl-61-40 satDNA family), that was previously identified and described $[17,18]$. The percentage of this satDNA family in the genome of $C$. album aggregate diploid species ranges from $0.25 \%$ (C. pamiricum) to $3.80 \%$ (C. acuminatum). It has been shown that this satDNA family is the most abundant and oldest component of the Chenopodium genome [18], but its origin is still unclear. In the present study, we aimed to infer the mutual relationships of the CficCl-61-40 satDNA family and TEs by analyzing the Illumina reads of 17 species of the $C$. album aggregate. We hypothesize that the origin of this family of repeats may be associated with the activity of one of the TEs.

\section{Results and discussion}

Association of TEs and CficCl-61-40 satDNA family arrays in the genome of $C$. acuminatum

The application of the Geneious Prime assembler [19] to the Illumina reads (genome coverage $0.4-0.9 x$ ) of seventeen diploid and polyploid C. album aggregate species (Table 1) resulted in the identification of CficCl-61-40 satDNA family arrays. The analysis of the relative positions of satDNAs and different TEs in the genome of $C$. acuminatum revealed cases of their colocalization with TEs (mainly with LTR retrotransposons), similar to the findings described by Heitkam et al. [20]. This is not surprising given that satDNA could be a target for TE insertions [21]. However, the comparative sequence analysis of CficCl-61-40 satDNA family arrays and retrotransposons revealed no significant similarities, and these cases could be regarded solely as insertions. In contrast to retrotransposons, several cases of interactions between the transposases (TPases) of DNA transposons (particularly those of putative CACTA-like elements) and CficCl-61-40 satDNA family arrays were revealed. Thus, in contig 22 of the C. acuminatum CficCl-61-40 satDNA family array of 94.4 monomers with a consensus sequence of $39 \mathrm{bp}$ was attached to the incomplete tnp 2 TPase domain of $115 \mathrm{bp}$, and the start monomer of the array was simultaneously a tnp 2 fragment of $41 \mathrm{bp}$ oriented in the same direction (Fig. 1a, Additional file 1). The similarity of the tnp2 fragment and the consensus sequence of the adjacent array was very high, at $89.7 \%$. Another case was found in contig 150 of the same species, in which a fragment of the tnp 2 TPase domain of 314 bp was attached to a CficCl-61-40 satDNA family array of 8.4 monomers. The similarity of the tnp2 fragment and the consensus sequence of the adjacent array was again $89.7 \%$. In contig 431 , a similar fragment of $t n p 2$ of $293 \mathrm{bp}$ and a CficCl-61-40 satDNA family array of 23.1 monomers were associated. The similarity of the tnp2 fragment and the consensus sequence of the adjacent array was $89.9 \%$. Finally, in contig 545 , a fragment of tnp 2 of 389 bp was associated with a CficCl-61-40 satDNA family array of 15.2 monomers, with the highest similarity of corresponding fragments reaching $94.9 \%$. Thus, in the genome of $C$. acuminatum, significant nucleotide similarities were found between monomers of the CficCl-61-40 satDNA family and the $\sim 40 \mathrm{bp}$ fragment of the tnp 2 domain, and it is possible to claim with a high probability that these monomers are derived from a similar region of their parent element, the $\sim 40 \mathrm{bp}$ fragment of $t n p 2$ of the putative CACTA-like transposon. Alternatively, but less likely, is a scenario in which minisatellites were captured by CACTA-like elements. Indeed, the capture of a single monomer or several monomers is possible, but this could not have led to the formation of a long associated 
Table 1 Chenopodium species used for the study, their genome composition, ploidy, genome size and geographical origin

\begin{tabular}{|c|c|c|c|c|c|}
\hline Species (accession number) & Genome composition [16] & Locality & Coordinates & Chr. No & $\begin{array}{l}\text { Genome } \\
\text { Size } \\
\text { Mbp }\end{array}$ \\
\hline C. acerifolium (316-1) & $B+D$ & Russian Federation, Velsk & $\begin{array}{l}\text { N } 61.066704 \\
\text { E } 42.095002\end{array}$ & $2 n=4 x=36$ & 2570 \\
\hline C. acuminatum (429-3) & $\mathrm{D}$ & China, Xinjiang, Altaj, Burqin & $\begin{array}{l}\text { N } 47.815500 \\
\text { E } 87.080028\end{array}$ & $2 n=2 x=18$ & 960 \\
\hline C. album (291-1) & $B+C+D$ & Czech Republic, Hrádek & $\begin{array}{l}\text { N } 48.781583 \\
\text { E } 16.261528\end{array}$ & $2 n=6 x=54$ & 3808 \\
\hline C. bryoniifolium (742-4) & A & $\begin{array}{l}\text { Russian Federation, Primorski Krai, } \\
\text { Nakhodka city district }\end{array}$ & $\begin{array}{l}\text { N } 42.88775 \\
\text { E } 132.722361\end{array}$ & $2 n=2 x=18$ & 2608 \\
\hline C. ficifolium (330-2) & B & Czech Republic, Slatina & $\begin{array}{l}\text { N } 50.226389 \\
\text { E } 14.210528\end{array}$ & $2 n=2 x=18$ & 1785 \\
\hline C. iljinii (433-9) & E & China, Xinjiang, Altaj, Hoboksar & $\begin{array}{l}\text { N } 46.541472 \\
\text { E } 85.358083\end{array}$ & $2 n=2 x=18$ & 1144 \\
\hline C. jenissejense (640) & $B+E$ & $\begin{array}{l}\text { Russian Federation, Verkhnekolymsky } \\
\text { raion, Popovka river mouth }\end{array}$ & $\begin{array}{l}N 64.646833 \\
\text { E } 151.640306\end{array}$ & $2 n=4 x=36$ & 2935 \\
\hline C. karoi (460) & $B+E$ & China, Xinjiang, Tumuxiukezhen & $\begin{array}{l}\text { N } 41.667306 \\
\text { E } 79.693528\end{array}$ & $2 n=4 x=36$ & 2929 \\
\hline C. luteorubrum (742-17) & $A+C+D$ & $\begin{array}{l}\text { Russian Federation, Primorski Krai, } \\
\text { Nakhodka city district }\end{array}$ & $\begin{array}{l}\text { N } 42.88775 \\
\text { E } 132.722361\end{array}$ & $2 n=6 x=54$ & 3247 \\
\hline C. novopokrovskyanum (463-3) & $C+D$ & China, Xinjiang, Tumuxiukezhen & $\begin{array}{l}\text { N } 41.667306 \\
\text { E } 79.693528\end{array}$ & $2 n=4 x=36$ & 1192 \\
\hline C. opulifolium (696-6) & $B+C+F$ & Iran, Kurdistan, Marivan & $\begin{array}{l}\text { N } 35.498461 \\
\text { E } 46.166946\end{array}$ & $2 n=6 x=54$ & 4421 \\
\hline C. pamiricum (830-3) & $\mathrm{E}$ & $\begin{array}{l}\text { Tajikistan, Gorno-Badakhshan } \\
\text { autonomous region, Murghob district }\end{array}$ & $\begin{array}{l}\text { N } 37.821667 \\
\text { E } 73.566667\end{array}$ & $2 n=2 x=18$ & 1154 \\
\hline C. sosnowskyi (788) & $A+G$ & $\begin{array}{l}\text { Iran, west Azerbaijan, Siah Cheshmeh } \\
\text { (Chaldoran) }\end{array}$ & $\begin{array}{l}\text { N } 39.065972 \\
\text { E } 44.386170\end{array}$ & $2 n=4 x=36$ & 2177 \\
\hline C. striatiforme (331-1) & $C+D$ & Czech Republic, Mělník & $\begin{array}{l}\text { N } 50.349528 \\
\text { E } 14.497444\end{array}$ & $2 n=4 x=36$ & 2029 \\
\hline C. strictum (380-5) & $C+D$ & Czech Republic, Prague & $\begin{array}{l}\text { N } 50.115964 \\
\text { E } 14.433326\end{array}$ & $2 n=4 x=36$ & 2022 \\
\hline C. suecicum (328-10) & B & Czech Republic, Švermov & $\begin{array}{l}\text { N } 50.176806 \\
\text { E } 14.105472\end{array}$ & $2 n=2 x=18$ & 1775 \\
\hline C. vulvaria (771-1) & $\mathrm{H}$ & Iran, Ardabil, Meshgin Shahr & $\begin{array}{l}\text { N } 38.405556 \\
\text { E } 47.694722\end{array}$ & $2 n=2 x=18$ & 924 \\
\hline
\end{tabular}

array of the CficCl-61-40 satDNA family, as we observed in the genome of $C$. acuminatum (see below). It is also essential to note that among all studied species, the genome of $C$. acuminatum exhibits the highest contents of the CficCl-61-40 satDNA family $(3.80 \%$ of the whole genome [18]).

A BLAST search for conserved domains identified the parental fragment as belonging to the TPase family member tnp2 pfam02992, a member of the cl29371 superfamily analogous to other tnp2 domains identified in several plants from different taxonomic groups [22] (Additional files 1, 2). Geneious Prime made it possible to detect the complete sequence of the tnp2 domain in the assembled contigs of the C. album aggregate species genomes (see Material and Methods), which show high similarity to known sequences from GenBank (Fig. 1b,
Additional file 2). The length of the tnp2 domains detected in the explored species was approximately $630 \mathrm{bp}$. Surprisingly, within the complete tnp2 domains (which are usually assembled in a single contig with DUF4216, DUF4218 and TPase-associated domains), we did not find the conserved TTTCATTTGA motif corresponding to the beginning of the tnp2 fragment parent of the CficCl-61-40 satDNA family array. Here, it is essential to note that in all cases recorded in the genome of $C$. acuminatum, only incomplete tnp2 domains of 115-389 bp could be recognized as parental to the CficCl-61-40 satDNA family array. Although these partial tnp2 domains were assigned to the pfam02992 family, they differed from the complete domains. Phylogenetic analysis showed them to form a separate branch derived from the internal regions (approximately positions 63-357, 


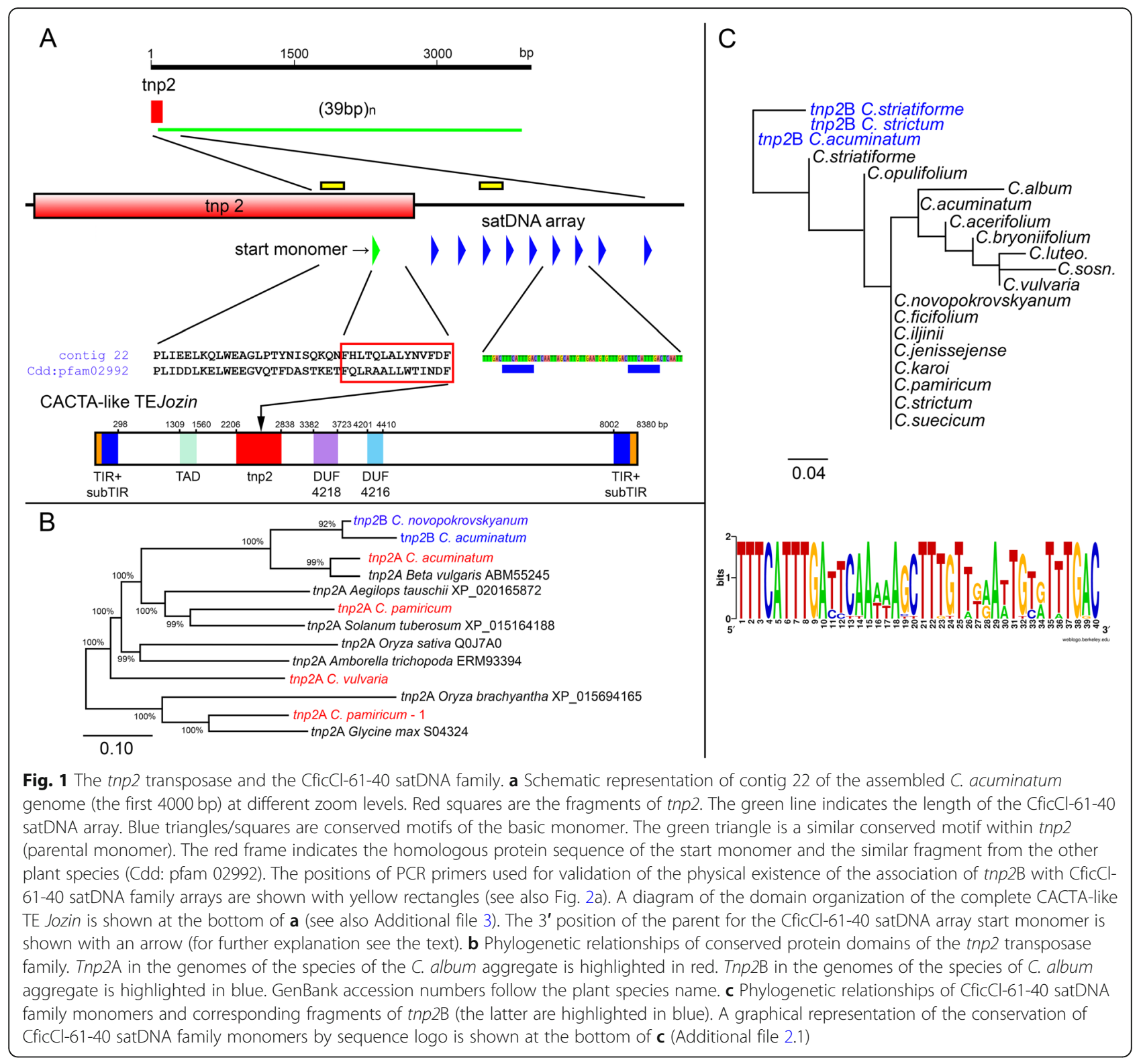

Fig. 1a, Additional file 2) of complete domains (Fig. 1b). These sequences could be assumed to be part of a putative novel short CACTA-like element but probably represent modified deletion derivatives of tnp2. These CACTA-related components of the Chenopodium genomes will hereafter be referred to as tnp2A (complete domains) and tnp2B (deletion derivatives parental to CficCl-61-40 satDNA family arrays).

\section{The tnp2B and CficCl-61-40 satDNA family array associations in genomes of other species of the C. album aggregate}

Searches in the genomes of the other studied species using the conserved motif specific to $\operatorname{tn} p 2 \mathrm{~B}$ (see Material and Methods) revealed six additional cases of tnp2B and
CficCl-61-40 satDNA family array links. The results are presented in Table 2 and Additional file 1. In the genomes of diploid C. ficifolium (B-genome), the tetraploids C. acerifolium (B- and D-genomes) and $C$. novopokrovskyanum (C- and D-genomes), and the hexaploid C. luteorubrum (A-, C- and D-genomes), fragments of tnp2B were identified, although without an association with CficCl-61-40 satDNA family arrays. In the other species, we did not find any tnp $2 \mathrm{~B}$ fragments, although domains of the tnp2A type were detectable, indicating the presence of CACTA transposons. Thus, we identified a complete putative CACTA element, which we refer to as Jozin, in the assembled genomes of $C$. pamiricum and C. sosnowskyi. The length of the element is approximately $8300 \mathrm{bp}$ with similarity between the 
Table 2 Presence and association of tnp2B and CficCl-61-40 satDNA family arrays (see also Additional file 1)

\begin{tabular}{|c|c|c|c|c|c|c|c|}
\hline Species & Genomes & $\operatorname{tnp} 2 B$ & $\begin{array}{l}\text { tnp2B+ } \\
\text { array }\end{array}$ & Contig No & $\begin{array}{l}\text { tnp2B } \\
\text { lengths }\end{array}$ & $\begin{array}{l}\text { Array } \\
\text { copy-numb }\end{array}$ & $\begin{array}{l}\text { Similarity } \\
\text { tnp2B-array }\end{array}$ \\
\hline C. acerifolium & $B+D$ & yes & no & - & - & - & - \\
\hline C. album & $B+C+D$ & yes & yes & 1990 & $579 \mathrm{bp}$ & 12.0 & $89.7 \%$ \\
\hline C. bryoniifolium & A & no & no & - & - & - & - \\
\hline C. ficifolium & B & yes & no & - & - & - & - \\
\hline C. iljinii & $E$ & no & no & - & - & - & - \\
\hline C. jenissejense & $B+E$ & no & no & - & - & - & - \\
\hline C. karoi & $B+E$ & no & no & - & - & - & - \\
\hline C. luteorubrum & $A+C+D$ & yes & no & - & - & - & - \\
\hline C. novopokrovskyanum & $C+D$ & yes & no & - & - & - & - \\
\hline C. opulifolium & $B+C+F$ & no & no & - & - & - & - \\
\hline C. pamiricum & $E$ & no & no & - & - & - & - \\
\hline C. sosnowskyi & $A+G$ & no & no & - & - & - & - \\
\hline \multirow[t]{2}{*}{ C. striatiforme } & $C+D$ & yes & yes & 541 & 134 bp & 54.2 & $89.8 \%$ \\
\hline & & & & 28,391 & 371 bp & 22.2 & $94.9 \%$ \\
\hline \multirow[t]{3}{*}{ C. strictum } & $C+D$ & yes & yes & 700 & $371 \mathrm{bp}$ & 27.9 & $90.0 \%$ \\
\hline & & & & 10,973 & 386 bp & 17.4 & $94.9 \%$ \\
\hline & & & & 11,346 & 341 bp & 27.1 & $80.0 \%$ \\
\hline C. suecicum & B & no & no & - & - & - & - \\
\hline C. vulvaria & $\mathrm{H}$ & no & no & - & - & - & - \\
\hline
\end{tabular}

tnp2A conserved domains of $92.9 \%$. The characteristics of the elements and their sequences are presented in Additional file 3 and Fig. 1a. Therefore, the distribution of tnp2B is uneven among the C. album aggregate species. All cases of tnp2B and CficCl-61-40 satDNA family array associations were connected with the D-genome (diploid C. acuminatum and polyploids harboring the Dgenome as a haplome, including C. album s. str., C. striatiforme and C. strictum), while most of the Bgenome-containing species (Tables 1 and 2) possessed fragments of tnp2B, but no association with the CficCl61-40 satDNA family arrays was found in silico.

These data raise a key question: is the discovered mechanism of satDNA family monomer formation unique to D- and, probably, B-genome lineages, or has it arisen independently and repeatedly during the evolution of a fairly broad group of Amaranthaceae species? The latter possibility can be indirectly supported by the interspecific similarity of basic monomers: a higher similarity indicates a greater probability that this mechanism exists or existed previously in a certain genome. Thus, to assess the homology of CficCl-61-40 satDNA family monomers in all investigated species, tandem repeat finder (TRF) was used, and consensus monomers were identified. Related HOR units [18] were excluded from the analysis, and monomers were aligned according to the conserved TTTCATTTGA motif, which was present in all monomers and corresponds to the beginning of the tnp2B parental fragment (Additional file 2). The corresponding parental fragments of tnp $2 \mathrm{~B}$ were also included. The similarity between the consensus monomers and parental tnp2B fragments ranged from 82.1 to $94.9 \%$ (Fig. 1c). Such high similarity makes it possible to suggest that CficCl-61-40 satDNA family monomers are derived from similar regions of the parental $\sim 40 \mathrm{bp}$ fragment of tnp2B in all studied genomes and that this event took place at least once in their evolutionary history. Moreover, the similar conserved motif present in Beta corolliflora minisatellites of $40 \mathrm{bp}$ (GenBank AJ288880.1) [23] extends the existence of the mechanism further back in time.

\section{Experimental validation of the computationally identified structures}

The generation of consensus sequences by assembling reads into contigs is problematic for satellites due to their tandem structure [18]. Thus, we aimed to confirm the existence of the physical counterparts of the computer-generated associations in the genome of $C$. acuminatum. The physical existence of the association of $t n p 2 \mathrm{~B}$ with CficCl-61-40 satDNA family arrays in the genome of $C$. acuminatum was supported by PCR screening, in which the forward, outward-facing primer corresponded to the conserved motif of tnp2B and the reverse primer to the CficCl-61-40 satDNA family array (Fig. 1a). The presence of a clear PCR band of the 


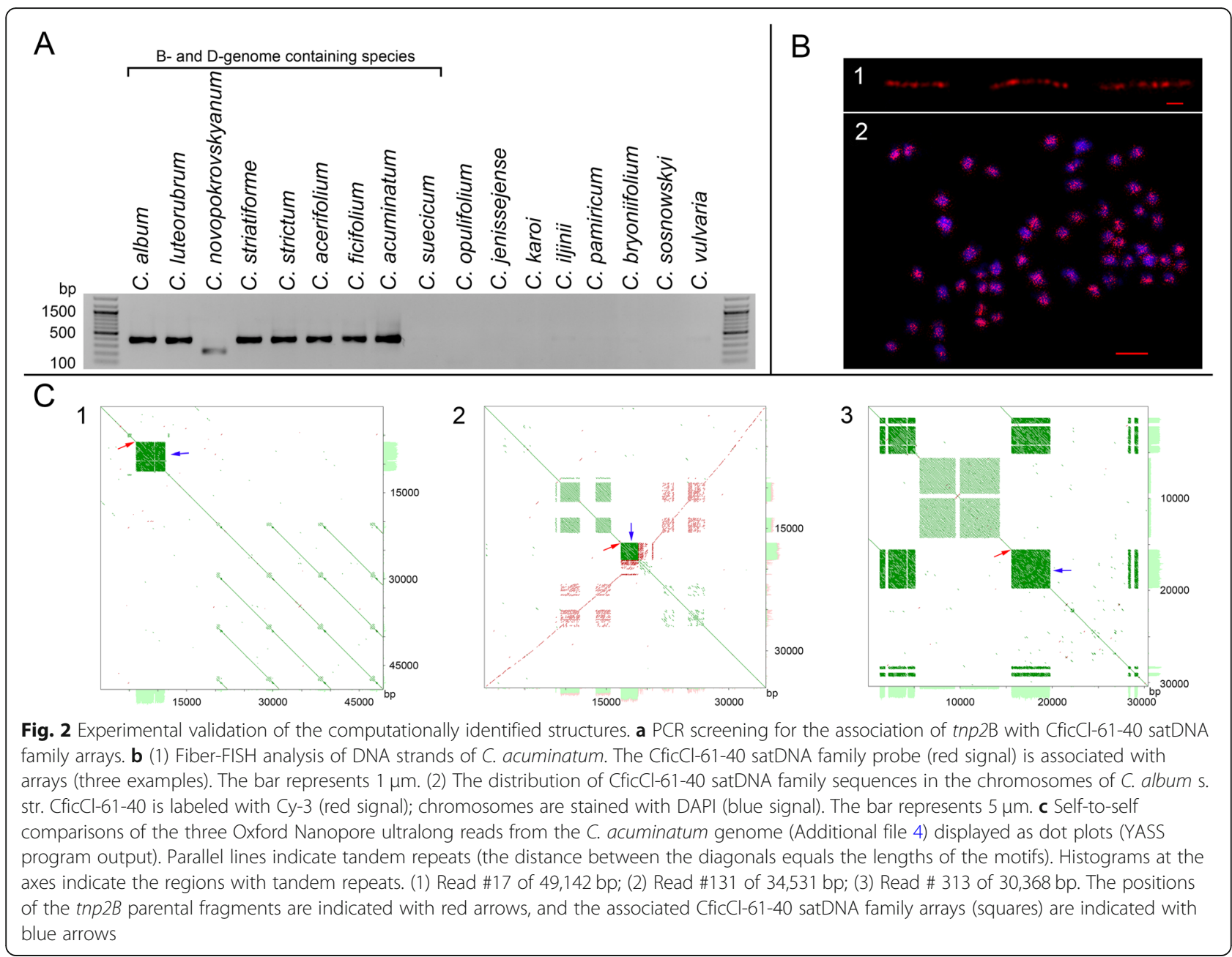

expected size ( $\sim 400 \mathrm{bp})$ confirmed the existence of associations between $t n p 2 \mathrm{~B}$ and $\mathrm{CficCl}-61-40$ satDNA family arrays in the genome of $C$. acuminatum (Fig. 2a). The cloning results for the PCR-amplified DNA fragments showed $95.8-96.3 \%$ similarity with the assembled reference sequence, which indicates the accuracy of the assembly algorithm. The PCR screening of the other species revealed putative associations of tnp2B and $\mathrm{CficCl}-61-40$ satDNA family arrays in the genomes of $C$. acerifolium, $C$. ficifolium, C. luteorubrum and C. novopokrovskyanum. In all of these species, the presence of the tnp2B fragment was also revealed based on the Illumina data. Moreover, we confirmed the association of tnp2B and CficCl-61-40 satDNA that was predicted in silico for $C$. album s. str., $C$. strictum and C. striatiforme (Fig. 2a). The remainder of the analyzed species showed negative results regarding the presence of a tnp2B and CficCl-61-40 satDNA association according to both PCR analysis (Fig. 2a) and the in silico screening of the Illumina data.

Another important question is whether the monomers of the CficCl-61-40 satDNA family form fairly long arrays in the genome or could be an artifact of the assembly process. To address this issue, we applied the fiber-FISH (fluorescent in situ hybridization) technique, in which fluorescent probes of CficCl-61-40 satDNA family monomers were hybridized directly to isolated DNA strands to detect a possible row of signals. The fiber-FISH experiments revealed long chains of signals (Fig. 2b.1), thus confirming the array structure of the CficCl-61-40 satDNA family.

One additional piece of evidence for $\operatorname{tnp} 2 B$ and CficCl-61-40 satDNA family array associations and the existence of long CficCl-61-40 satDNA family arrays was provided by an alternative assembly-free approach for the analysis of ultralong Oxford Nanopore $(\mathrm{ON})$ reads. In Fig. 2c, self-to-self comparisons of several ultralong $\mathrm{ON}$ reads from the $C$. acuminatum genome (Additional file 4) displayed as dot plots (YASS program output) are shown. Parallel lines indicate tandem repeats in which the distance between the diagonals is equal to the lengths of the motifs. This type of analysis confirmed both the tnp2B-satDNA association and the complicated 
structure of CficCl-61-40 satDNA family arrays [18]. ON sequencing also allowed the determination of the real length of the CficCl-61-40 satDNA family array that follows the tnp2B fragment. For $\mathrm{ON}$ read 17 , the array consisted of $\sim 125$ tandemly arranged monomers $(5.0 \mathrm{~kb})$; for read 131, it was $\sim 48$ monomers $(1.9 \mathrm{~kb})$; and for read 313, it was $\sim 105$ monomers $(4.2 \mathrm{~kb}$ ) (Fig. 2c).

\section{Concluding remarks}

Recently, we described the evolution of the CficCl-61-40 satDNA family in the genomes of several diploid Chenopodium species [18]. This family is a major component of the Chenopodium satellitome with a panchromosomal distribution (Fig. 2b.2). It has been hypothesized that this family of minisatellites could have arisen in the ancestral forms of Chenopodioideae and then been transmitted vertically during evolution, with subsequent divergence of both the nucleotide composition and length of the basic monomer. Definitely, this type of CficCl-61-40 satDNA family evolution exists in the Chenopodium genomes, and several tandem repeats have diverged significantly. For example, clone 12-13p from the genomes of C. quinoa (GenBank: HM641822.1) did not show similarity to any existing GenBank sequences at the time when it was discovered [24]. However, with the subsequent arrival of new data [18], similarities were found between clone 12-13p and the recently identified HOR unit from the $C$. acuminatum genome, which belongs to the CficCl-61-40 satDNA family (GenBank: MH257681.1 - MH257684.1). Thus, the $12-13 p$ repeat could be attributed to a distantly diverged derivative of the CficCl-61-40 satDNA family. Now, it can be argued that along with vertical transmission, in at least some of the studied genomes, there is an ongoing process of CficCl-61-40 satDNA family monomer formation from the TPases of putative CACTA-like elements. This phenomenon is similar to that found in Arabidopsis by Kapitonov and Jurka [11], in which one of the $\mathrm{En} / \mathrm{Spm}$-like transposons (Atenspm) generates satellite arrays, and it is the internal region of the En/Spmlike transposon (as in our case) that is specifically involved in array formation. Therefore, the pool of CficCl61-40 satDNA family sequences is continuously replenished. The specific mechanism of CficCl-61-40 satDNA family array formation from the fragment of tnp $2 \mathrm{~B}$ requires further investigation but is probably associated with CACTA-like transposon activity in the genome. TE proliferation is a dynamic process that may occur repeatedly over short evolutionary timescales. The active autonomous (intact) DNA elements in trans may influence non-autonomous (deletion derivatives) elements as long as they retain the cis-acting target sequences [25], and CACTA deletion derivatives may often generate regions of tandem repeated DNA such as the $A f a$ and
TM-2 repeats in Triticeae [26]. Thus, there are two prerequisites for new CficCl-61-40 satDNA family monomer formation: (i) the presence of an active complete CACTA-like element and (ii) the presence of deletion derivatives of the tnp2B type. High similarity between parental tnp2B fragments and related satDNA monomers and their interposition in the genome may indicate that CACTA-like transposons have recently been active in the genomes of several C. album aggregate species. Thus, two pools of the CficCl-61-40 satDNA family could coexist: an ancestral pool and a newly formed pool.

What might be the consequences of the continuous generation of novel repeats of a certain type in the genome? The most salient point is that the continuous replenishment of genomes with new identical (because of tnp2 domains high conservation) CficCl-6140 satDNA family monomers may lead to an increase in satellitome similarity between species, which may in turn increase the possibility of interspecific hybridization [27]. The second important point is that in the genomes of several Chenopodium species, CACTA-like elements could be transpositionally active, with associated consequences [2, 28]. CACTAlike elements can cycle between an active and an inactive state [29]. At one extreme, an element may change frequently between states during plant development, whereas at the other extreme, the inactive state is stably inherited through many plant generations, and reactivation is not much more frequent than spontaneous mutation [2]. Thus, the question of which Chenopodium lineages still show activity of CACTA-like elements remains to be answered, which will significantly contribute to understanding Chenopodium genome evolution.

\section{Materials and methods}

Plant material, DNA extraction, library preparation and Illumina sequencing

For the preparation of the DNA libraries, plants of the following species were used: $C$. acerifolium $C$. acuminatum, C. album s. str., C. bryoniifolium, $C$. ficifolium, C. iljinii, C. jenissejense, C. karoi, C. luteorubrum, C. novopokrovskyanum, C. opulifolium, C. pamiricum, C. sosnowskyi, C. striatiforme, C. strictum, C. suecicum, and C. vulvaria (Table 1 ). DNA was extracted from fresh leaves using the DNeasy Plant Mini Kit (Qiagen) according to the manufacturer's instructions. For in situ hybridization experiments, the tips of the young, fine roots of C. album s. str. Were collected and fixed as described previously [15], and then stored until use. For all analyzed accessions, the DNA ploidy level was checked by flow cytometry as described previously [30]. One individual per species 
was used for library preparation and NGS. The details of library preparation and Illumina sequencing were as described in Belyayev et al. [18]. The Illumina data have been deposited in the NCBI Sequence Read Archive as BioProject PRJNA634444.

\section{Genome assembly, the search for repeatome elements and data processing}

For the processing of Illumina NGS data and the identification of the colocalization of TEs and CficCl-61-40 satDNA family arrays in the genomes of all investigated species, Geneious Prime software version 2019.2.1 (https://www.geneious.com) was used [19]. The advantage of this assembler is that it produces large contigs. De novo assembly was carried out with medium-low sensitivity, which is the best option for large numbers (e.g., 100,000 or more) of Illumina sequencing reads. The main satellite was identified using a short conserved motif as a query. This motif, generated on the basis of our previous research [18], was TTTCATTTGA. In contigs with CficCl-61-40 satDNA family arrays, a search for TEs was carried out using BLAST searches for conserved domains (https://www.ncbi.nlm.nih.gov/Structure/cdd/wrpsb.cgi) [31]. TRF (https://tandem.bu.edu/ trf/trf.html) analysis [32] allowed the determination of the consensus monomers of the CficCl-61-40 satDNA family. The described algorithms were applied to the genomes of each species separately.

Genomes were scanned for the presence of the tnp2A and tnp $2 \mathrm{~B}$ TPases in individual contigs with the aid of the conserved motifs. The tnp2A TATAACTTGCCTCC TT motif was first detected in the genomes of C. acuminatum and C. vulvaria. The position of the motif covered the region from 159 to $175 \mathrm{bp}$ of the $\sim 630 \mathrm{bp}$ tnp 2 domain. For $\operatorname{tn} p 2 \mathrm{~B}$, the conserved GGCTGGGTTACC motif was detected $20 \mathrm{bp}$ upstream from the beginning of the tnp2B fragment parental to the CficCl-61-40 satDNA family array. The latter motif was missing in the full domains. Scanning was performed by using the "search for motifs" command of Geneious software with a one-nucleotide maximum mismatch for tnp $2 \mathrm{~A}$ and a two-nucleotide maximum mismatch for tnp2B.

For the reconstruction of phylogenetic relationships among the analyzed monomers and TPases, multiple alignments were performed with ClustalW [33]. The phylogenetic relationships among the sequences were then reconstructed from the pairwise distance matrix [34]. The distance matrix thus obtained could be used to construct a phylogenetic tree via the minimum evolution method. The construction of the phylogenetic tree was performed in the MEGA program (Fig. 1b, c) [35]. The sequence logo was produced using the publicly available online tool: https://weblogo.berkeley.edu/logo.cgi [36].
PCR screening for the association of tnp2B with CficCl-6140 satDNA family arrays

Primer pairs were designed for the PCR screening of the association of tnp2B with CficCl-61-40 satDNA family arrays, in which the $5^{\prime}$ primer was an outward-facing tnp2B-specific primer based on the conserved GGCTGG GTTACC motif, and the 3' primer corresponded to the beginning of the CficCl-61-40 satDNA family array (contig 22 of C. acuminatum) (Fig. 1a). If an association of tnp2B with CficCl-61-40 satDNA family arrays physically exists in the genome, we would expect to obtain a combined fragment of approximately $400 \mathrm{bp}$ consisting of sequences from both the tnp2B sequence and the $\mathrm{CficCl}$ 61-40 satDNA family array. If such an association does not exist and the suggested association is an artifact of the assembly process, no PCR product will be observed. The oligonucleotide sequences of the PCR primers were as follows: 5' tnp2B-specific primer, GGCTGGGTTA CCGACTTACA; 3' CficCl-61-40 satDNA array-specific primer, TCAAACATGTACATCCAGCCA. As a template, we used the total DNA of all investigated species (Fig. 2a). PCR was performed in $25 \mu \mathrm{l}$ reactions containing $1 \mathrm{x}$ TopBio Plain PP Master Mix (TopBio), each primer at $0.2 \mathrm{mM}$ and $5 \mathrm{ng}$ of genomic DNA. The cycling conditions were as follows: $5 \mathrm{~min}$ at $95^{\circ} \mathrm{C}$, followed by 35 cycles of $95^{\circ} \mathrm{C}$ for $30 \mathrm{~s}$, the sequence-specific annealing temperature $\left(55^{\circ} \mathrm{C}\right)$ for $30 \mathrm{~s}$ and $72{ }^{\circ} \mathrm{C}$ for $1.5 \mathrm{~min}$, and a final extension at $72{ }^{\circ} \mathrm{C}$ for $15 \mathrm{~min}$. The PCR results were verified in a $1 \%$ agarose gel. The PCR products of clusters were excised from the gels, cloned and sequenced at Eurofins Genomics (Konstanz, Germany) according to standard protocols.

\section{FISH procedure}

To detect the chromosomal distribution of the CficCl61-40 satDNA family in the chromosomes of C. album s. str., FISH was performed. Root tips were pretreated in $0.002 \mathrm{M} 8$-hydroxyquinolin for $3 \mathrm{~h}$ in the dark and fixed in $3: 1(\mathrm{v} / \mathrm{v}) 100 \%$ ethanol:acetic acid. The fixed root meristems were thoroughly washed in water and enzyme buffer $(10 \mathrm{mM}$ citrate buffer at $\mathrm{pH} 4.6)$ and partially digested in $0,3 \%(\mathrm{w} / \mathrm{v})$ cytohelicase, pectolyase and cellulase (Sigma, St. Louis, MS, USA) at $37^{\circ} \mathrm{C}$ for $3 \mathrm{~h}$, followed by several washes in water. The material in a water drop was carefully transferred to a grease-free microscope slide, and the cells were spread as previously described [18]. Fiber-FISH slides were prepared from the total DNA of $C$. acuminatum according to the technique described by Schwarzacher and Heslop-Harrison [37].

FISH experiments were performed with the CficCl-61$40 \mathrm{X}-1$ clone [18] as a probe, which was labeled with Cy3 (Amersham, Amersham, UK) according to a standard oligo labeling protocol. FISH was performed in a ThermoBrite programmable temperature-controlled 
slide processing system at $63^{\circ} \mathrm{C}$ for $3 \mathrm{~h}$. The slides were stained with DAPI, mounted in antifade mountant (Vector Laboratories, Peterborough, UK) and examined and photographed on a Zeiss Axio Imager.Z2 microscope system.

\section{Oxford Nanopore sequencing and ultralong read analysis for the association of tnp2B and the CficCl-61-40 satDNA family}

For Oxford Nanopore sequencing, the DNA of C. acuminatum was used. The DNA was fragmented by pipetting. The sequencing libraries were prepared from $1 \mu \mathrm{g}$ of the partially fragmented DNA using an SQK-LSK109 Ligation Sequencing Kit (Oxford Nanopore Technologies) following the manufacturer's protocol. The DNA was treated with $2 \mu \mathrm{l}$ of NEBNext FFPE DNA Repair Mix and $3 \mu \mathrm{l}$ of NEBNext Ultra II End-prep enzyme mix in a $60 \mu \mathrm{l}$ volume that also included $3.5 \mu \mathrm{l}$ of FFPE and $3.5 \mu \mathrm{l}$ of End-prep reaction buffers (New England Biolabs). The reaction was performed at $20^{\circ} \mathrm{C}$ for $5 \mathrm{~min}$ and $65^{\circ} \mathrm{C}$ for $5 \mathrm{~min}$, followed by purification using a $1 \mathrm{x}$ volume of AMPure XP beads (Beckman Coulter). Subsequent steps, including adapter ligation using NEBNext Quick T4 DNA Ligase and library preparation for sequencing, were performed according to the provided protocols. The whole library was loaded into the FLOMIN106 R9.4 flow cell and sequenced for $20 \mathrm{~h}$.

A search for tnp $2 B$ was performed with the conserved GGCTGGGTTACC motif among the longest (>30 kbp) $\mathrm{ON}$ reads of the $C$. acuminatum genome. For the analysis of selected ultralong ON reads, the YASS genomic similarity tool was used, which enables searches of tandem repeat organization (http://bioinfo.lifl.fr/yass/yass. php) $[18,38]$.

\section{Supplementary information}

Supplementary information accompanies this paper at https://doi.org/10. 1186/s13100-020-00219-7.

Additional file 1: Contig showing the association of thp2B and $\mathrm{CficCl}$ 61-40 satDNA family arrays.

Additional file 2: 1 . Consensus monomers of the CficCl-61-40 satDNA family and corresponding fragments of tnp2 for species of the C. album aggregate. 2. TPase domains of putative CACTA-like transposons detected in the genomes of the species of the $C$. album aggregate in comparison with similar domains of other species.

Additional file 3: Characteristics of the putative CACTA element Jozin from the genomes of $C$. pamiricum and C. sosnowskyi.

Additional file 4: Three $O N$ reads from the $C$. acuminatum genome with association of tnp2B and CficCl-61-40 satDNA family arrays.

\section{Abbreviations}

DUF: Domain of unknown function; FISH: Fluorescent in situ hybridization; HOR: High order repeats; NGS: Next-generation sequencing; ON: Oxford Nanopore sequencing; satDNA: Satellite DNA; TAD: Transposase associated domain; TEs: Transposable elements; TPase: Transposase; TRF: Tandem repeats finder.

\section{Authors' contributions}

$A B$ conceived the idea for the study. BM and KK collected the plant material. $A B, K K, J$, and $M J$ performed or supervised the wet lab work. $A B, V M$, and $K K$ analyzed the data. $A B, B M, V M$, and KK wrote the manuscript and Supporting Information. The authors read and approved the final manuscript.

\section{Funding}

This work was supported by the Czech Science Foundation (grant No 2020286S) and as part of a long-term research development project RVO 67985939. The funding agencies had no role in the design of the study and collection, analysis, and interpretation of data.

\section{Availability of data and materials}

Please contact author for data requests for the datasets used and/or analyzed during the current study including the original fasta sequencing files. All annotations files and generated output data sets corresponding to number of contigs and/or reads mapping putative CACTA-like element Jozin and $\mathrm{CficCl}-61-40$ satDNA family arrays are included in this published article (and its supplementary information files).

Ethics approval and consent to participate

Not applicable.

\section{Consent for publication}

Not applicable.

\section{Competing interests}

The authors declare that they have no competing interests.

\section{Author details}

${ }^{1}$ Czech Academy of Sciences, Institute of Botany, Zámek 1, CZ-252 43 Průhonice, Czech Republic. ${ }^{2}$ Faculty of Environmental Sciences, Czech University of Life Sciences Prague, Kamýcká 129, 16500 Praha, Suchdol, Czech Republic.

Received: 24 March 2020 Accepted: 19 June 2020

Published online: 26 June 2020

\section{References}

1. Mehrotra S, Goyal V. Repetitive sequences in plant nuclear DNA: types, distribution, evolution and function. Genom Proteom Bioinformat. 2014;12: 164-71. https://doi.org/10.1016/j.gpb.2014.07.003.

2. Kunze R, Saedler H, Lonning W-E. Plant transposable elements. Adv Bot Res. 1997;27:331-470.

3. Bennetzen JL. The contributions of retroelements to plant genome organization, function and evolution. Trends Microbiol. 2000;4:347-53.

4. Koonin EV. Evolution of genome architecture. Int J Biochem Cell Biol. 2009; 41:298-306.

5. Biscotti MA, Olmo E, Heslop-Harrison JS. Repetitive DNA in eukaryotic genomes. Chromosom Res. 2015;23:415-20. https://doi.org/10.1007/s10577015-9499-z.

6. Satović E, Vojvoda Zeljko T, Luchetti A, Mantovani B, Plohl M. Adjacent sequences disclose potential for intra-genomic dispersal of satellite DNA repeats and suggest a complex network with transposable elements. BMC Genomics. 2016;17:997. 27919246

7. Martienssen RA. Maintenance of heterochromatin by RNA interference of tandem repeats. Nat Genet. 2003;35:213-4. https://doi.org/10.1038/ng1252.

8. Kloc A, Martienssen R. RNAi, heterochromatin and the cell cycle. Trends Genet. 2008;24:511-7. https://doi.org/10.1016/j.tig.2008.08.002.

9. Garrido-Ramos MA. SatDNA in plants: more than just rubbish. Cytogenet Genome Res. 2015;146:153-70. https://doi.org/10.1159/000437008.

10. Meštrović N, Mravinac B, Pavlek M, Vojvoda-Zeljko T, Šatović E, Plohl M. Structural and functional liaisons between transposable elements and satellite DNAs. Chromosom Res. 2015;23:583-96. https://doi.org/10.1007/ s10577-015-9483-7.

11. Kapitonov W, Jurka J. Molecular paleontology of transposable elements from Arabidopsis thaliana. Genetica. 1999:107:27-37.

12. Sharma A, Wolfgruber TK, Presting GG. Tandem repeats derived from centromeric retrotransposons. BMC Genomics. 2013;14:142. https://doi.org/ 10.1186/1471-2164-14-142 
13. Chu G-L, Mosyakin SL, Clemants SE. Chenopodiaceae. In: Wu Z, et al., editors. Flora of China 5. St. Louis: Missouri Botanical Garden Press; 2003. p. $351-414$.

14. Habibi F, Vít P, Rahiminejad M, Mandák B. Towards a better understanding of the C. album aggregate in the Middle East: a karyological, cytometric and morphometric investigation. J Syst Evol. 2018;56:231-42. https://doi.org/10. 1111/jse.12306.

15. Mandák B, Krak K, Vít P, Pavlíková Z, Lomonosova MN, Habibi F, Lei W, Jellen EN, Douda J. How genome size variation is linked with evolution within Chenopodium sensu lato. Perspect Plant Ecol Evol System. 2016;23:18-32.

16. Mandák B, Krak K, Vít P, Lomonosova MN, Belyayev A, Habibi F, Wang L, Douda J, Storchova H. Hybridization and polyploidization within the Chenopodium album aggregate analyzed by means of cytological and molecular markers. Mol Phylogenet Evol. 2018;129:189-201.

17. Kolano B, Gardunia BW, Michalska M, Bonifacio A, Fairbanks D, Maughan PJ, et al. Chromosomal localization of two novel repetitive sequences isolated from the Chenopodium quinoa Willd genome. Genome. 2011;54:710-7. https://doi.org/10.1139/G11-035.

18. Belyayev A, Josefiová J, Jandová M, Kalendar R, Krak K, Mandák B. Natural history of a satellite DNA family: from the ancestral genome component to species-specific sequences, Concerted and Non-Concerted Evolution. Int J Mol Sci. 2019;20:5. https://doi.org/10.3390/ijms20051201.

19. Kearse M, Moir R, Wilson A, Stones-Havas S, Cheung M, Sturrock S, et al. Geneious basic: an integrated and extendable desktop software platform for the organization and analysis of sequence data. Bioinformatics. 2012;28: 1647-9. https://doi.org/10.1093/bioinformatics/bts199.

20. Heitkam T, Weber B, Walter I, Ost C, Schmidt T. Satellite DNA landscapes after allotetraploidisation of quinoa (Chenopodium quinoa) reveal unique a and B subgenomes. Plant J. 2020. https://doi.org/10.1111/tpj.14705.

21. Kejnovský E, Michalovova M, Steflova P, Kejnovska I, Manzano S, Hobza R, et al. Expansion of microsatellites on evolutionary young $Y$ chromosome. PIOS ONE. 2013;8:e45519. https://doi.org/10.1371/journal.pone.0045519.

22. Majorek KA, Dunin-Horkawicz S, Steczkiewicz K, Muszewska A, Nowotny M, Ginalski K, Bujnicki JM. The RNase H-like superfamily: new members, comparative structural analysis and evolutionary classification. Nucl Acids Res. 2014;42:4160-79.

23. Gao D, Schmidt T, Jung C. Molecular characterization and chromosomal distribution of species-specific repetitive DNA sequences from Beta corolliflora, a wild relative of sugar beet. Genome. 2000;43:1073-80.

24. Orzechowska M, Majka M, Weiss-Schneeweiss H, Kovařík A, Borowska-Zuchowska $\mathrm{N}$, Kolano B. Organization and evolution of two repetitive sequences, 18-24J and 12-13P, in the genome of Chenopodium (Amaranthaceae). Genome. 2018;61:64352. https://doi.org/10.1139/gen-2018-0044.

25. McClintock B. The control of gene action in maize. Brookhaven Symp Biol. 1965:18:162-84.

26. Wicker T, Guyot R, Yahiaoui N, Keller B. CACTA transposons in Triticeae. A diverse family of high-copy repetitive elements. Plant Physiol. 2003;132:52-63.

27. Belyayev A. Bursts of transposable elements as an evolutionary driving force. J Evol Biol. 2014;27:2573-84. https://doi.org/10.1111/jeb.1251.

28. Raskina O, Belyayev A, Nevo E. Activity of the en/Spm-like transposons in meiosis as a base for chromosome repatterning in a small, isolated, peripheral population of Aegilops speltoides Tausch. Chromosom Res. 2004; 12:153-61.

29. McClintock $B$. The contribution of one component of a control system to versatility of gene expression. Carnegie Institution of Washington Year Book. 1971;70:5-17.

30. Vít P, Krak K, Trávníček P, Douda J, Lomonosova MN, Mandák B. Genome size stability across Eurasian Chenopodium species (Amaranthaceae). Bot J Linn Soc. 2016;182:637-49.

31. Marchler-Bauer A, Bo Y, Han L, He J, Lanczycki CJ, Lu S, et al. DD/SPARCLE: functional classification of proteins via subfamily domain architectures. Nucl Acids Res. 2017:45(D1):D200-3. https://doi.org/10.1093/nar/gkw1129.

32. Benson G. Tandem repeats finder: a program to analyze DNA sequences. Nucl Acids Res. 1999:27:573-80

33. Vinga S, Almeida J. Alignment-free sequence comparison - a review. Bioinformatics. 2003;19:513-23.

34. Edgar RC. MUSCLE: a multiple sequence alignment method with reduced time and space complexity. BMC Bioinformatics. 2003;5:113.

35. Kumar S, Stecher G, Li M, Knyaz C, Tamura K. MEGA X: Molecular evolutionary genetics analysis across computing platforms. Molec Biol Evol. 2018;35:1547-9. https://doi.org/10.1093/molbev/msy096.
36. Crooks GE, Hon G, Chandonia JM, Brenner SE. WebLogo: A sequence logo generator. Genome Res. 2004;14:1188-90. https://doi.org/10.1101/gr.849004.

37. Schwarzacher T, Heslop-Harrison JS. Practical in situ hybridization. Oxford: BIOS Scientific Publishers; 2000.

38. Noe L, Kucherov G. YASS: enhancing the sensitivity of DNA similarity search. Nucl Acids Res. 2005;33:W540-3.

\section{Publisher's Note}

Springer Nature remains neutral with regard to jurisdictional claims in published maps and institutional affiliations.
Ready to submit your research? Choose BMC and benefit from:

- fast, convenient online submission

- thorough peer review by experienced researchers in your field

- rapid publication on acceptance

- support for research data, including large and complex data types

- gold Open Access which fosters wider collaboration and increased citations

- maximum visibility for your research: over $100 \mathrm{M}$ website views per year

At BMC, research is always in progress.

Learn more biomedcentral.com/submissions 